Vinícius Lopes Rodrigues

\title{
Visualização de Dados Geográficos Vetoriais sobre Terrenos em Multi-Resolução
}

Dissertação apresentada como requisito parcial para obtenção do grau de Mestre pelo Programa de Pós-graduação em Informática do Departamento de Informática da PUC-Rio

Orientador: Prof. Waldemar Celes Filho 


\title{
Vinícius Lopes Rodrigues
}

\section{Visualização de Dados Geográficos Vetoriais sobre Terrenos em Multi-Resolução}

\begin{abstract}
Dissertação apresentada como requisito parcial para obtenção do grau de Mestre pelo Programa de Pós-graduação em Informática do Departamento de Informática do Centro Técnico Científico da PUC-Rio. Aprovada pela Comissão Examinadora abaixo assinada.
\end{abstract}

\author{
Waldemar Celes Filho \\ Orientador \\ Departamento de Informática - PUC-Rio
}

Prof. Marcelo Gattass

Departamento de Informática - PUC-Rio

Prof. Luis Henrique Figueiredo Instituto de Matemática Pura e Aplicada - IMPA

Dr. Marcelo Tílio Monteiro de Carvalho

Pesquisador - Tecgraf/PUC-Rio

Prof. José Eugenio Leal

Coordenador do Centro Técnico Científico da PUC-Rio

Rio de Janeiro, 21 de Dezembro de 2009 
Todos os direitos reservados. É proibida a reprodução total ou parcial do trabalho sem autorização da universidade, do autor e do orientador.

\section{Vinícius Lopes Rodrigues}

Graduou-se em Ciência da Computação pela Universidade Federal de Viçosa. Trabalhou junto ao Tecgraf - PUC-Rio na área de Software de Informação Geográfica e Algoritmos Geométricos. Desenvolveu como projeto de mestrado uma visualização conjunta de dados geográficos vetoriais com modelos tridimensionais de terreno.

Ficha Catalográfica

Rodrigues, Vinícius

Visualização de Dados Geográficos Vetoriais sobre Terrenos em Multi-Resolução / Vinícius Lopes Rodrigues; orientador: Waldemar Celes Filho. — 2009

v., 62 f: il. ; $29,7 \mathrm{~cm}$

1. Dissertação (Mestrado em Informática) - Pontifícia Universidade Católica do Rio de Janeiro, Rio de Janeiro, 2009

Inclui bibliografia

1. Informática - Teses. 2. Correção Perspectiva. 3. Sistemas de Informação Geográfica. 4. Terrenos em Multiresolução. 5. Simplificação de Dados Geográficos. I. Celes Filho, Waldemar. II. Pontifícia Universidade Católica do Rio de Janeiro. Departamento de Informática. III. Título. 


\section{Agradecimentos}

Ao meu orientador Waldemar Celes por ter me orientado com a paciência e atenção de um grande mestre.

Ao CNPq pelo incentivo financeiro durante o trabalho.

Ao Tecgraf pelo apoio desde o início do mestrado.

Aos colegas de Tecgraf por proporcionar o melhor ambiente de trabalho possível.

Aos meus familiares pelos momentos de alegria, além dos vários exemplos de luta e superação.

Aos meus amigos que tanto me ouviram durantes esses anos, e assim me deram forças para continuar e alcançar esse objetivo.

À minha irmã Thalita pelas risadas e broncas.

Aos meus pais por terem sempre se esforçado tanto para que tivesse o melhor.

Ao meu amigo e irmão Valente por me fazer acreditar que eu era capaz e estava próximo de conseguir o título. Desde a primeira semana de aula. 


\section{Resumo}

Rodrigues, Vinícius; Celes Filho, Waldemar. Visualização de Dados Geográficos Vetoriais sobre Terrenos em Multi-Resolução. Rio de Janeiro, 2009. 62p. Dissertação de Mestrado — Departamento de Informática, Pontifícia Universidade Católica do Rio de Janeiro.

O presente trabalho visa integrar a representação gráfica de dados vetoriais encontrados em Sistemas de Informação Geográfica (SIG) com a visualização de modelos tridimensionais de terrenos em multi-resolução. A multiresolução do terreno implica em dificuldades para a renderização dos dados vetoriais de forma coerente, uma vez que estes dados, em geral, só armazenam informações bi-dimensionais. As soluções mais comumente empregadas usam mapeamento convencional de texturas, trazendo uma qualidade visual baixa. Para melhorar a qualidade da imagem gerada, adotamos o mapeamento de textura com correção perspectiva, similar ao que tem sido empregado para geração de mapas de sombras. Além disso, para obter eficiência na visualização de dados vetoriais complexos, apresentamos um algoritmo de multi-resolução destes dados. Na solução proposta, a simplificação dos dados vetoriais é desacoplada da multi-resolução utilizada no modelo tridimensional do terreno. Com isso, nossa solução pode ser adotada em diferentes visualizadores de terreno. Apresentamos resultados que ilustram a eficiência da solução proposta.

\section{Palavras-chave}

Correção Perspectiva. Sistemas de Informação Geográfica. Terrenos em Multi-resolução. Simplificação de Dados Geográficos. 


\section{Abstract}

Rodrigues, Vinícius; Celes Filho, Waldemar(Advisor). Vectorial geographic data visualization on multi-resolution terrain. Rio de Janeiro, 2009. 62p. M.Sc Dissertation - Departamento de Informática, Pontifícia Universidade Católica do Rio de Janeiro.

This work aims to integrate the graphical representation of vectorial data found in Geographic Information Systems (GIS) and the visualization for multi-resolution 3D terrain models. The multi-resolution approach on terrain rendering brings some difficulties on the process of rendering the vectorial data in a consistent manner, since these data, in general, only store bi-dimensional information. The solutions most commonly used are based on conventional texture mapping, resulting in low visual quality. In order to improve image quality, we adopt texture mapping with perspective correction, in a way similar to the techniques used for shadow map generation. Besides, willing to obtain efficiency on complex vectorial data visualization, a multi-resolution algorithm of these data is presented. In our proposed solution, vectorial data simplification is decoupled from the multi-resolution technique used on the tri-dimensional terrain model. Thus, our solution can be used with different terrain viewers. We present results that illustrate the efficiency of the proposed solution.

\section{Keywords}

Perspective Correction. Geographic Information System. Multiresolution Terrain. Geographic Data Simplification. 


\section{Sumário}

1 Introdução $\quad 11$

1.1 Falhas Visuais no Desenho Vetorial sobre Terrenos 13

$\begin{array}{lll}1.2 & \text { Objetivos e Contribuições } & 14\end{array}$

$\begin{array}{lll}1.3 & \text { Organização do Texto } & 16\end{array}$

2 Trabalhos Relacionados $\quad 17$

$\begin{array}{lll}2.1 & \text { Terrenos em Multi-Resolução } & 17\end{array}$

2.2 Correção Perspectiva em Mapas de Sombra 19

2.3 Desenho de Dados Vetoriais sobre Terrenos 22

2.4 Simplificação de Geometrias 26

3 Texturização com Dados Geográficos $\quad 28$

3.1 Organização dos Dados Geográficos 28

3.2 Desenho das Primitivas Geográficas 29

3.3 Transformação e Aplicação da Textura 30

3.4 Matrizes de Transformação 31

4 Geração Eficiente da Textura $\quad 36$

4.1 Simplificação dos Dados Geográficos 36

4.2 Hierarquia de Simplificação 36

4.3 Qualidade e Coerência da Simplificação 38

4.4 Escolha do Nível de Detalhe dos Dados 40

5 Resultados $\quad 43$

5.1 Eficiência da simplificação de dados geográficos 43

5.2 Qualidade da Correção Perspectiva 47

5.3 Navegação e Escolha de Blocos na Hierarquia 51

5.4 Visualização Conjunta Mapa 2D com Terreno 3D 54

6 Conclusão e Trabalhos Futuros $\quad 56$

6.1 Trabalhos Futuros $\quad 56$

$\begin{array}{ll}\text { Referências Bibliográficas } & 59\end{array}$ 


\section{Lista de figuras}

1.1 Visualização de um mapa em um SIG tradicional 12

1.2 Exemplo de desenho de dados vetoriais sobre o terreno do Google Earth

1.3 Exemplo de desenho de dados vetoriais sobre o terreno do Nasa World Wind

1.4 Exemplo de desenho de dados vetoriais sobre o terreno do ArcGIS Explorer

2.1 Visualização do LOD da dissertação de Magalhães (Mag05) 19

2.2 Definição dos eixos do espaço da luz 20

2.3 Passos do algoritmo do Light Space 21

2.4 Gráfico do perfil do terreno para um segmento 23

2.5 Efeitos de undersampling e oversampling, respectivamente 25

2.6 Resultado do trabalho de Schneider et al. (Extraída de (Sch05)) 26

3.1 Cálculo dos valores para a matriz sem correção perspectiva 32

3.2 Heurística da Bounding Box para o cálculo da área da textura 33

4.1 Simplificação de polígonos utilizando o algoritmo de Douglas-Peucker 37

4.2 Incoerência topológica devido à simplificação 39

4.3 Fragmentação que garante a coerência entre fronteiras 40

5.1 Mapa de ferrovias da Ásia 44

5.2 Mapa de rios permanentes do Brasil $\quad 45$

5.3 Mapa de fronteiras de municípios do Brasil 46

5.4 Comparação da visualização sem e com correção perspectiva. Rio 49

5.5 Comparação da visualização sem e com correção perspectiva. Brasil 50

5.6 Gráfico comparativo da simplificação dos dados geográficos. Caso de Teste 1

5.7 Screenshot de pontos notáveis do gráfico. Caso de Teste 1

5.8 Porcentagem dos vértices totais efetivamente utilizados. Caso de Teste 1

5.9 Gráfico comparativo da simplificação dos dados geográficos. Caso de Teste 2

5.10 Porcentagem dos vértices totais efetivamente utilizados. Caso de Teste 2

5.11 Comparação da taxa de quadros por segundo entre as duas visualizações

6.1 Configuração do aliasing de projeção (Extraída de (Sch05)) 


\section{Lista de tabelas}

5.1 Configuração do computador dos testes 43

5.2 Características dos dados geográficos de teste. 43

5.3 Resultados dos testes com o tema de Ferrovias da Ásia 45

5.4 Resultados dos testes com o tema de Rios Permanentes do Brasil 46

5.5 Resultados dos testes com o tema de Fronteiras dos Munícipios do Brasil

5.6 Características dos dados geográficos no teste Rio de Janeiro 47

5.7 Características dos dados geográficos no teste Brasil 48

5.8 Taxa de quadros por segundo média para cada visualização 54 
Nihil est ab omni Parte beatum.

Nada é belo visto de todos os pontos de vista.

Quintus Horatius Flaccus 